\title{
Effects of Graded Exercises Integrated with Education on Physical Fitness, Exercise Self- Efficacy, and Activity Levels in People with Spinal Cord Injury: A Quasi-Experimental Study Protocol
}

\author{
Hafifi Hisham $^{1,2}$, Maria Justine ${ }^{1}$, Hafez Hussain ${ }^{2}$, Nazirah Hasnan ${ }^{3}$, Haidzir Manaf ${ }^{1,4}$ \\ ${ }^{1}$ Centre for Physiotherapy, Faculty of Health Sciences, Universiti Teknologi MARA, Puncak Alam Campus, Selangor, Malaysia \\ ${ }^{2}$ Pusat Rehabilitasi PERKESO, Melaka, Malaysia \\ ${ }^{3}$ Department of Rehabilitation Medicine, Faculty of Medicine, Universiti Malaya, Kuala Lumpur, Malaysia \\ ${ }^{4}$ Clinical and Rehabilitation Exercise Research Group, Faculty of Health Sciences, Universiti Teknologi MARA, Puncak Alam Campus, Selangor, Malaysia
}

Study Design: A quasi-experimental single-blinded study.

Purpose: To investigate the effects of 'graded exercise integrated with education' on physical fitness, exercise self-efficacy (ESE), and physical activity (PA) levels among subacute and chronic wheelchair-dependent paraplegia patients.

Overview of Literature: Most of the chronic spinal cord injury (SCI) patients had low physical fitness due to a sedentary lifestyle and lack of ESE after discharge from a rehabilitation program. Education may encourage them to engage with exercise to regain and maintain their physical fitness. However, there is a lack of research to support the effects of exercise integrated with education after an SCl.

Methods: A total of 44 participants will be assigned to either the experimental group (graded exercise integrated with education) or active control (conventional physical therapy). The experimental group will receive graded strength and aerobic exercise training according to their progression criteria. They will attend an education program during and after the rehabilitation program. The control group will only receive conventional physical therapy during their in-rehabilitation program. This study will be conducted during a period of 16 weeks, consisting of 8 weeks of in-rehabilitation and 8 weeks post-rehabilitation. Statistical analysis will be performed using the IBM SPSS ver. 21.0 (IBM Corp., Armonk, NY, USA) at a significance level of $p \leq 0.05$.

Results: The primary outcome measures will be upper-limb isokinetic strength, isometric grip strength, and cardiorespiratory fitness. The secondary outcomes will be ESE and PA levels.

Conclusions: An intervention that combines exercise training and education may be warranted to enhance the physical fitness, ESE, and PA levels in SCI patients. This trial was registered with ClinicalTrials.gov (NCT03420170).

Keywords: Health education; Physical activity; Physical fitness; Self efficacy; Spinal cord injuries; Spine

Received Jul 12, 2018; Revised Nov 26, 2018; Accepted Dec 13, 2018

Corresponding author: Haidzir Manaf

Centre for Physiotherapy, Faculty of Health Sciences, Universiti Teknologi MARA, Puncak Alam Campus, 42300 Puncak Alam, Selangor, Malaysia

Tel: +60-3-3258-4599, Fax: +60-3-3258-4599, E-mail: haidzir5894@puncakalam.uitm.edu.my 


\section{Introduction}

Injury to the spinal cord due to a traumatic or nontraumatic event is a devastating condition. A spinal cord injury (SCI) may result in the loss of motor function and sensation below the vertebral level of the injury [1]. The autonomic body systems for bowel, bladder, thermoregulation, and cardiovascular function may also become dysregulated [1]. Individuals with SCI generally have a low level of physical activity (PA) after discharge [2] from a rehabilitation program, and this may lead to further decline in physical and mental well-being. The decline in physical health status may increase the risk of secondary complications such as cardiovascular diseases [3], metabolic disorders [4,5], and obesity [4].

A previous systematic review found positive outcomes of exercise on physical capacity such as strength, body composition, and functional performance [6]. However, a more recent review conducted by Bochkezanian et al. [7] found limited evidence to conclude any significant effect of strengthening combined with aerobic exercises on physical fitness, muscle strength, and function. It has also been recommended that psychological or behavioral intervention may boost exercise self-efficacy (ESE) and PA levels following discharge from a rehabilitation program [8].

Several studies demonstrated positive effects of PA integrated with behavorial or education programs on the levels of PA. For instance, a study conducted by Pelletier et al. [9] found that sustaining participation and adherence of individuals with SCI improved PA. This finding is supported by Nooijen et al. [10] who found that a combination of PA integrated with a behavior program increased physical fitness and PA levels. However, this study did not highlight the effects of such intervention on the physical fitness and ESE [10]. Therefore, an investigation on the effects of graded exercise integrated with education on physical fitness, ESE, and PA levels is needed to establish the scientific evidence for the clinical rehabilitation of SCI patients.

\section{Materials and Methods}

\section{Research design}

This is a single-blinded, nonrandomized (quasi-experimental) study comparing two groups of SCI patients: experimental group (graded exercise integrated with education) and active control group (conventional physical therapy). This study will be conducted in a vocational rehabilitation clinic (SOCSO Tun Razak Rehabilitation Centre, Melaka, Malaysia). This trial was registered with ClinicalTrials.gov (NCT03420170). This study protocol was approved by UITM Research Ethics Committee (REC/138/18).

\section{Participants}

The inclusion criteria for participation in the study are patients with traumatic or non-traumatic SCI who are wheelchair-dependent paraplegics (American Spinal Injury Association Impairment Scale A, B, C), female or male, aged between $18-55$ years, with subacute ( $>4$ months since injury), or chronic SCI ( $>1$ year since injury). Participants will be excluded if they are pregnant, have progressive neurological diseases, depression, mental disorder, unhealed fractures, heart diseases, severe pain, frequent autonomic dysreflexia, and hypotension as diagnosed by a resident medical doctor. The participants are also not eligible to participate if they are unable to understand or read instructions in Malay or English language.

\section{Study procedure}

Participants will give written, informed consent after being briefed regarding study procedures, and will then be randomly allocated to either the experimental or active control group. Each group will have the same number of upper and lower thoracic SCI participants to control for any confounding effects. The experimental group will receive graded exercise training integrated with education for 8 weeks while in the rehabilitation program. After 8 weeks, participants will be discharged from the rehabilitation program, but will continue receiving education intervention once every 4 weeks, for a duration of 8 weeks (total of two post-rehabilitation education sessions). The educational sessions will be conducted by the main researcher (a trained physiotherapist) supervised by a resident clinical psychologist. The control group will receive conventional physical therapy intervention such as unstructured strengthening, stretching and aerobic exercises, bed mobility and transfer training for 8 weeks, and will receive no education after discharge from the rehabilitation program.

The assessment of physical fitness will be conducted 


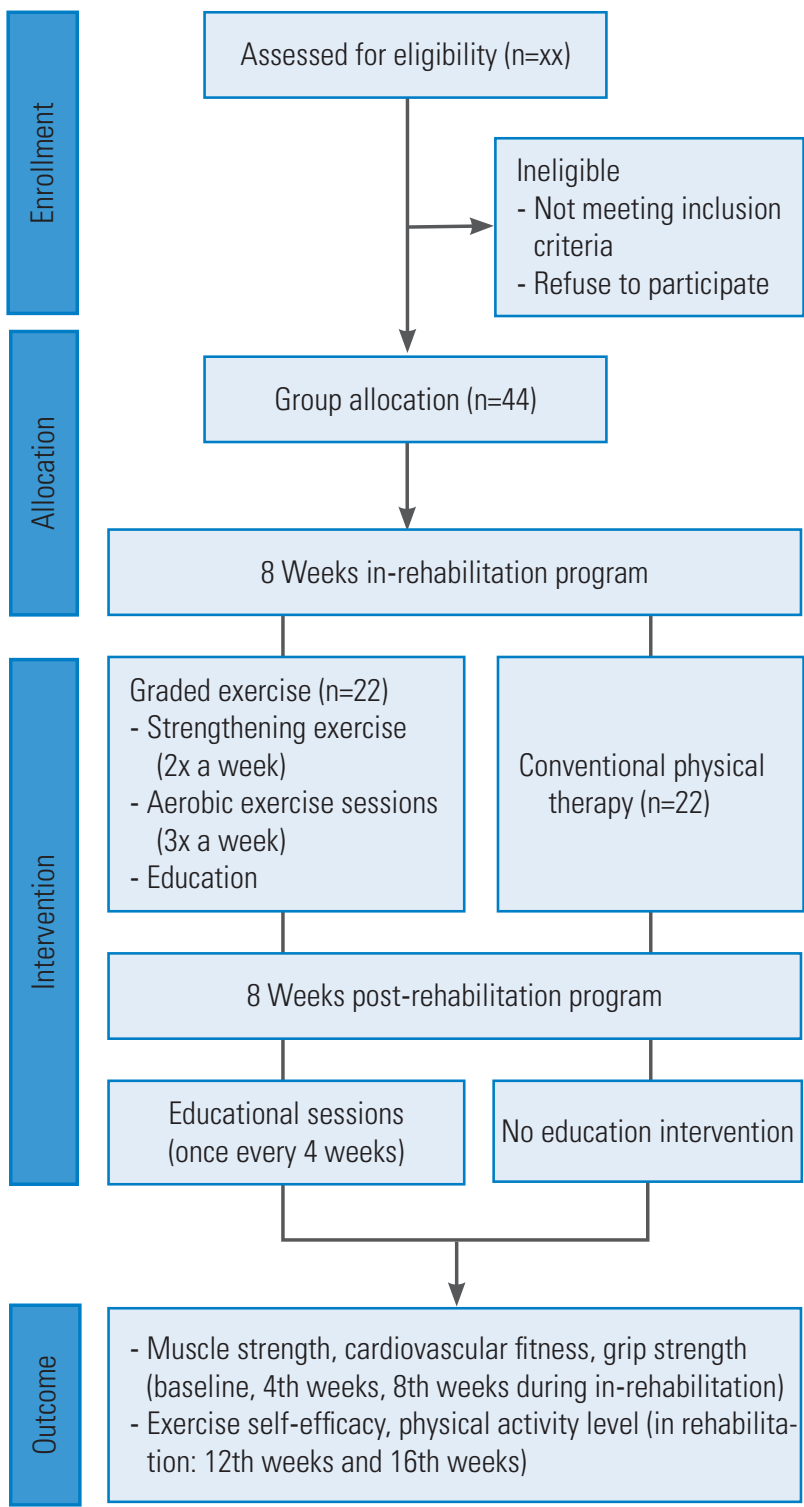

Fig. 1. The study flow chart.

during the in-rehabilitation program at baseline, the 4th week, and the 8th week. The evaluation of ESE and PA levels will be conducted during the in-rehabilitation program at baseline, 4th week, 8th week, and post-rehabilitation at 12th and 16th week. The study flow chart is shown in Fig. 1.

\section{Interventions}

The graded exercise training sessions will consist of strength and aerobic exercises on alternate days [11]. The training will be supervised by a trained physiotherapist, and the intensity of exercise will be increased weekly if participants meet their progression criteria.

The objective of strengthening exercises is to maintain muscle force and power production for functional activities such as transfer and wheelchair propelling [4]. The strengthening exercise will follow the recently published recommendations [11], which suggested that an individual with SCI should perform at least 4-5 types of strengthening exercises including the major muscles for shoulder stabilizers, internal and external rotators for three sets, 8-12 repetitions each set, and 2 minutes of rest between sets. These exercises should be performed at least 2 days a week, alternately. Training will be conducted in a circuit, utilizing a closed-chain multi-gym machine for vertical chest press, butterfly press, vertical row, wide latissimus pull down, internal and external rotations. Dumbbell exercises will focus on triceps push, biceps curl, shoulder flexion, and abduction. The intensity will be set between moderate to high intensity, starting from $50 \%$ of the 1 repetition measure $[12,13]$. A full repetition is defined as a 6-second movement pattern, with approximately 3-second concentric and 3-second eccentric contraction phases [14]. The intensity will progress weekly if the participants are able to complete the sets without signs of tremor or compensatory movement.

The purpose of the aerobic exercise is to provide sufficient physical stress on the cardiovascular, pulmonary, and metabolic systems of the body to initiate an overload and training effect which is important for daily life function [4]. The protocol for the aerobic circuit training was modified from a previous study [5] to meet the recommendation from a recent position statement [11], which suggested that an individual with SCI should perform at least 20 minutes of moderate to vigorous aerobic exercise (12-15 on the Borg scale) for 3 days alternately in a week. The circuit will consist of wheelchair propelling exercise (30 $\mathrm{m}$ loop), punching bag boxing, and arm ergometry hand-cycling (MOTOmed Viva 2 Movement Therapy Trainer; Reck-Technik GmbH \& Co., Betzenweiler, Germany). The circuit will start with 3.5 minutes of exercise followed by 1.5 minutes of rest (21 cumulative minutes of exercise) and will gradually increase to 4.5 minutes of exercise followed by 30 seconds of rest (27 cumulative minutes of exercise). Therefore, a total of 30 minutes will be spent for each level of exercise. Two rounds of three different exercises will be performed at each level (six cumulative exercises). The participants will progress to the next level when they are able to complete the exercise minutes and complete the exertion goal at their current level without pain or overbearing fatigue, defined as fatigue that 
interferes with activities of daily living or regular therapy, as reported by the participants. The exercise intensity will be monitored via a heart rate monitor and on a Borg scale of perceived exertion. Participants will be required to perform 2 minutes hand-cycling without resistance for warm-up and cool down. Table 1 shows the levels and intensity of the graded exercise.

The educational intervention aimed to increase ESE and PA levels will be conducted in a small closed group of a maximum of four persons, taught by the researcher and supervised by a resident clinical psychologist for 2 hours each week for a total of 8 weeks during the inrehabilitation program and once in 4 weeks for a total of 8 weeks after discharge from the rehabilitation program via a telephone motivational counseling. The educational syllabus will consist of: [1] formulation of action planning and goal settings, [2] strategies to become active, [3] coping and barrier anticipation, [4] the type of PA and exercise for manual wheelchair, [5] benefits of PA and exercise for manual wheelchair, and [6] the risk and safety during exercise. The educational syllabus and framework were developed using the guidelines from the 'appraisal of guideline research and evaluation' [15].

\section{Outcome measures}

The primary outcome is physical fitness, which includes upper-limb isokinetic strength (peak torque, peak torque normalized by body weight, total work, and average power), hand grip isometric strength, and cardiorespiratory fitness (6-minute wheelchair push test [6-MWPT]). The rationale of physical fitness as the primary outcome measure is to investigate physical performance, which influences the functional potential to overcome the overload on upper limb during transfer and wheelchair propelling [16], to assess upper-limb impairment, to determine the efficacy of treatment [17], and predict fitness levels of participants, which thus will influence exercise and functional capacity in daily life [18]. The secondary outcome is the ESE and PA levels, which will be measured using the ESE scale (ESES) and Physical Activity Scale for Individual with Disability (PASIPD), respectively. The rationale for ESE and PA levels as secondary outcomes is to predict the internal or personal barrier toward exercise [19], and to

Table 1. The levels and intensity of the graded aerobic exercise

\begin{tabular}{|c|c|c|c|c|}
\hline Level & Value on Borg RPE & $\begin{array}{c}\text { Total minutes at indicated value } \\
\text { on/of Borg RPE }\end{array}$ & Exercise (min) & Rest (min) \\
\hline 1 & $12-14$ & 21 & 3.5 & 1.5 \\
\hline 2 & $12-14$ & 24 & 4 & 1 \\
\hline 3 & $14-16$ & 24 & 4 & 1 \\
\hline 4 & $14-16$ & 27 & 4.5 & 0.5 \\
\hline 5 & $14-18$ (1 min at RPE value of $14+1$ min at RPE value of 18$) \times 2$ & 24 & 4 & 1 \\
\hline
\end{tabular}

$\mathrm{RPE}$, rating perceived exertion

Table 2. The upper-limb strength assessment protocol

\begin{tabular}{|c|c|c|c|}
\hline Movement & Total range of motion & Intensity & Position \\
\hline Elbow: bilateral flexion and extension & $90^{\circ}$ & $\begin{array}{l}60 / \text { sec and } 80 / \text { sec ( } 5 \text { repetitions) } \\
10 \text { sec rest } \\
\text { 5-Minute rest between change }\end{array}$ & $\begin{array}{l}\text { Elbow: } 35^{\circ} \text { flexion } \\
\text { Hand: neutral }\end{array}$ \\
\hline Shoulder: bilateral IR and ER & $70^{\circ}\left(\right.$ ER: $0^{\circ}-40^{\circ}$, IR: $\left.0^{\circ}-30^{\circ}\right)$ & $\begin{array}{l}60^{\circ} / \mathrm{sec} \text { and } 180^{\circ} / \mathrm{sec} \text { ( } 5 \text { repetitions) } \\
300^{\circ} / \mathrm{sec} \text { ( } 10 \text { repetitions) } \\
1 \text { Minute of rest for each movement }\end{array}$ & $\begin{array}{l}\text { Shoulder: shoulder abductor: } 45^{\circ} \\
\text { shoulder flexor: } 30^{\circ} \\
\text { Elbow: } 35^{\circ} \text { flexion } \\
\text { Hand: neutral }\end{array}$ \\
\hline $\begin{array}{l}\text { Shoulder: bilateral flexor, extensor, } \\
\text { abductor and adductor }\end{array}$ & $\begin{array}{l}105^{\circ} \text { (flexor: } 0^{\circ}-70^{\circ} \text {, extensor: } \\
0^{\circ}-35^{\circ} \text { ) } \\
55^{\circ} \text { (abductor and adductor: } \\
15^{\circ}-70^{\circ} \text { ) }\end{array}$ & $\begin{array}{l}30,60 \text {, and } 120 / \mathrm{sec} \\
1 \text { Minute of rest for each movement }\end{array}$ & $\begin{array}{l}\text { Elbow: } 35^{\circ} \text { flexion } \\
\text { Hand: neutral }\end{array}$ \\
\hline
\end{tabular}

IR, internal rotation; ER, external rotation. 
predict the risk of secondary complications [20] after injury, such as cardiovascular diseases, type II diabetes mellitus, obesity, depression as well as lifestyle behavior [21]. About 1 week will be allocated to assess the participants using the mentioned outcome measures.

To measure muscle strength, an isokinetic dynamometric assessment [22] will be completed using a Biodex isokinetic system-3 (Biodex Medical Systems Inc., Shirley, NY, USA) to quantify strength via (1) peak torque, (2) total work (J), and (3) average power in watts. The peak torque will be normalized to body weight in N.m. ${ }^{\mathrm{kg}-1}$ and quantified to compare the strength between participants with different morphology. The test movements include shoulder internal and external rotations, shoulder flexion, extension, abduction and adduction, as well as elbow extension and flexion. The amplitudes, angular velocities, and range of the movement to be tested will replicate the shoulder kinematic parameters frequently performed during wheelchair propulsion and sitting pivot transfers among individuals with paraplegia [16]. Table 2 shows the assessment protocol of the measurements.

The protocol for isometric hand grip assessment will follow the standard procedures published by Sisto and Dyson-Hudson [23]. The score will be the average of three measurements with a 15-second rest period between trials to avoid fatigue. The duration of isometric exercise will range from 3 to 5 seconds. Strength will be measured in both hands using the Jamar hand grip dynamometer (J A Preston Corp., New York, NY, USA). The grade of the resistance of the hand grip tool is set at level 3 difficulty. The posture must be in the upright position and rested on the back support of the wheelchair. The wheelchair needs to be stabilized by pulling the brake to avoid wobbling or unexpected movements. Hands will be placed on a table with the shoulder of the tested side abducted to $30^{\circ}$ and the elbow flexed to $90^{\circ}$ with the forearm positioned in a half supinated position while the hand dynamometer is gripped in the hand. The fist will be off the table and supported by the palm of the assessor's hand.

Cardiorespiratory fitness will be measured using the 6-MWPT as recommended by Solanki et al. [24] and Cowan et al. [18]. The 6MWPT was found to be valid, reliable, sensitive, and sufficient to measure cardiorespiratory fitness among sedentary individuals with SCI [18], particularly when the resources such as qualified personnel and sophisticated instruments are unavailable or are limited. The course will be a $30-\mathrm{m}$ loop marked by two cones spaced $15 \mathrm{~m}$ apart with $2.8 \mathrm{~m}$ on either end to allow for turning. There will be two $180^{\circ}$ turns to complete the $30-\mathrm{m}$ loop. Participants will be instructed to propel themselves as far as possible on the propulsion course and will be advised that they can slow or stop at any point during the testing. The participants will be allowed to practice for 2 minutes before the actual test begins. They will be advised to keep a velocity akin to pushing in a grocery store. A standardized lightweight wheelchair $(14 \mathrm{~kg})$ will be used for testing all participants. The distance traveled in 6 minutes will be computed by multiplying the number of completed laps by $15 \mathrm{~m}$ and adding the distance traveled in the last lap. The distance traveled in the turns will not be measured, in accordance with the standard protocol of the assessment.

The ESES is a valid and reliable tool, and highly consistent internally to measure the level of ESE among individuals with SCI [25]. The ESES consists of 10 items to assess the level of self-confidence to perform regular PA and exercise among people with SCI. The higher the score, the greater the perceived ESE. The ESES will be translated into Malay language to suit the participants who do not understand English.

The PASIPD will be used to measure the levels of PA among people with SCI. The PASIPD was found to be reliable and valid to measure the PA levels among people with disabilities [26]. The PASIPD will capture information regarding leisure, household, and work related PA over the preceding 7 days. It will assess five distinct dimensions of PA including home repair, lawn and garden work, housework, vigorous sport and recreation, moderate sport and recreation, and occupation and transportation. The PASIPD has the potential to provide in-depth information about the degree of activity in the form of metabolic equivalents (METs hr/day), or descriptive information regarding various activities each individuals can perform. The scoring will be based on the average hours per day for each item, then multiplied by a MET value associated with the intensity of the activity and summing over items 2 through 13 . The scores can range from 0 (no activity) to $>100$ METs hr/day (very high). The PASIPD will be translated to Malay language to suit the population in Malaysia.

\section{Results}

Study outcomes will be analyzed quantitatively to present the means, standard deviations, absolute values, percent- 
ages, and 95\% confidence intervals of relevant variables. The results will be displayed in tables and figures. The significance levels will be set at $p \leq 0.05$. The KolmogorovSmirnov test will be used to check the normality of the related variables. Repeated measures analysis of variance will be conducted to compare dependent variables for within and between groups. All measures will be presented with their respective effect sizes and 95\% confidence interval. All data analysis will be conducted using IBM SPSS statistical software ver. 21.0 (IBM Corp., Armonk, NY, USA).

\section{Discussion}

The main objective of this study is to determine the effects of graded exercise integrated with education intervention on physical fitness, ESE, and levels of PA among wheelchair-bound individuals with SCI. It is hypothesized that participants in the experimental group may achieve significant improvements in physical fitness, ESE, and PA levels compared to the active control group, consistent with previous reviews $[6,7]$. However, more studies are needed to investigate the effects of exercises combined with education on functional cardiorespiratory fitness performance, such as the 6-MWPT and isokinetic strength tests. These tests replicate the functional range of motion during wheelchair propulsion and pivot transfer. A positive outcome in the cardiorespiratory fitness is important in enhancing physical fitness and endurance during activities which require high demand of oxygen supply, such as wheelchair propelling to go to work, sports, and recreation [16]. The significant improvements in strength between the angle of $35^{\circ}$ of shoulder extension and $70^{\circ}$ of shoulder flexion may enhance the performance of wheelchair propulsion [16], whereas, about $15^{\circ}-60^{\circ}$ of abduction and adduction is important for independent transfer performance [16]. The strength performance and agonist-antagonist balance of the shoulder internal and external rotation are important for prevention of injury, particularly for wheelchair users [16]. The education intervention is important to provide knowledge and selfconfidence for SCI patients to perform self-exercise and PA for as long as possible. The confidence is important for individuals with SCI to be knowledgeable of exercises and to cope and adapt exercises to stay active at all times and places despite issues of accessibility, facilities, and environmental barriers. Therefore, graded exercise integrated with education could be useful as an intervention for SCI rehabilitation as it may improve outcomes compared to conventional physical therapy. However, more studies need to be conducted to support these recommendations.

\section{Conclusions}

It is hypothesized that the graded exercises integrated with education may provide a superior effect compared to the conventional physical therapy on physical fitness, exercise self-efficacy, and PA levels after a 16-week intervention. Therefore, findings from this study may contribute to scientific knowledge and practical strategies to enhance and maintain the physical fitness in people with SCI, particularly after discharge from a rehabilitation program.

\section{Conflict of Interest}

No potential conflict of interest relevant to this article was reported.

\section{Acknowledgments}

The authors thank the Ministry of Education, Malaysia, for funding the research project through the Fundamental Research Grant Scheme (Ref. no., 600-IRMI/FRGS 5/3 [014/2017]) and the Research Management Institute, Universiti Teknologi MARA for the administrative support.

\section{References}

1. Harvey LA. Physiotherapy rehabilitation for people with spinal cord injuries. J Physiother 2016;62:4-11.

2. Van den Berg-Emons RJ, Bussmann JB, Haisma JA, et al. A prospective study on physical activity levels after spinal cord injury during inpatient rehabilitation and the year after discharge. Arch Phys Med Rehabil 2008;89:2094-101.

3. Nooijen CF, de Groot S, Postma K, et al. A more active lifestyle in persons with a recent spinal cord injury benefits physical fitness and health. Spinal Cord 2012;50:320-3.

4. Sisto SA, Evans N. Activity and fitness in spinal cord injury: review and update. Curr Phys Med Rehabil Rep 2014;2:147-57.

5. Tawashy AE, Eng JJ, Krassioukov AV, Miller WC, Sproule S. Aerobic exercise during early rehabilitation for cervical spinal cord injury. Phys Ther 
2010;90:427-37

6. Hicks AL, Martin Ginis KA, Pelletier CA, Ditor DS, Foulon B, Wolfe DL. The effects of exercise training on physical capacity, strength, body composition and functional performance among adults with spinal cord injury: a systematic review. Spinal Cord 2011;49:1103-27.

7. Bochkezanian V, Raymond J, de Oliveira CQ, Davis GM. Can combined aerobic and muscle strength training improve aerobic fitness, muscle strength, function and quality of life in people with spinal cord injury?: a systematic review. Spinal Cord 2015;53:418-31.

8. Ashford S, Edmunds J, French DP. What is the best way to change self-efficacy to promote lifestyle and recreational physical activity?: a systematic review with meta-analysis. Br J Health Psychol 2010;15:26588.

9. Pelletier CA, Latimer-Cheung AE, Warburton DE, Hicks AL. Direct referral and physical activity counselling upon discharge from spinal cord injury rehabilitation. Spinal Cord 2014;52:392-5.

10. Nooijen CF, Stam HJ, Bergen MP, et al. A behavioural intervention increases physical activity in people with subacute spinal cord injury: a randomised trial. J Physiother 2016;62:35-41.

11. Tweedy SM, Beckman EM, Geraghty TJ, et al. Exercise and sports science Australia (ESSA) position statement on exercise and spinal cord injury. J Sci Med Sport 2017;20:108-15.

12. Pelletier CA, Totosy de Zepetnek JO, MacDonald MJ, Hicks AL. A 16-week randomized controlled trial evaluating the physical activity guidelines for adults with spinal cord injury. Spinal Cord 2015;53:363-7.

13. Totosy de Zepetnek JO, Pelletier CA, Hicks AL, MacDonald MJ. Following the physical activity guidelines for adults with spinal cord injury for 16 weeks does not improve vascular health: a randomized controlled trial. Arch Phys Med Rehabil 2015;96:156675.

14. Nash MS, van de Ven I, van Elk N, Johnson BM. Effects of circuit resistance training on fitness attributes and upper-extremity pain in middle-aged men with paraplegia. Arch Phys Med Rehabil 2007;88:70-5.
15. Brouwers MC, Kho ME, Browman GP, et al. AGREE II: advancing guideline development, reporting and evaluation in health care. CMAJ 2010;182:E839-42.

16. Dehail P, Gagnon D, Noreau L, Nadeau S. Assessment of agonist-antagonist shoulder torque ratios in individuals with paraplegia: a new interpretative approach. Spinal Cord 2008;46:552-8.

17. Innes EV. Handgrip strength testing: a review of the literature. Aust Occup Ther J 1999;46:120-40.

18. Cowan RE, Callahan MK, Nash MS. The 6-min push test is reliable and predicts low fitness in spinal cord injury. Med Sci Sports Exerc 2012;44:1993-2000.

19. Scelza WM, Kalpakjian CZ, Zemper ED, Tate DG. Perceived barriers to exercise in people with spinal cord injury. Am J Phys Med Rehabil 2005;84:576-83.

20. Jorgensen S, Iwarsson S, Lexell J. Secondary health conditions, activity limitations, and life satisfaction in older adults with long-term spinal cord injury. PM R 2017;9:356-66.

21. O'Halloran PD, Blackstock F, Shields N, et al. Motivational interviewing to increase physical activity in people with chronic health conditions: a systematic review and meta-analysis. Clin Rehabil 2014;28:115971.

22. Freitas PS, Serenza FD, Santana TS, Manoel LS, Riberto M. Isokinetic analysis of rotator cuff musculature of sedentary wheelchair users and basketball athletes with spinal cord injury. J Phys Med Rehabil Disabil 2017;3:017.

23. Sisto SA, Dyson-Hudson T. Dynamometry testing in spinal cord injury. J Rehabil Res Dev 2007;44:123-36.

24. Solanki R, Chaudhari P, Bhise A. Cardio respiratory fitness testing in spinal cord injury patients using 6 minute push test. Healthline J Indian Assoc Prev Soc Med 2016;7:60-3.

25. Kroll T, Kehn M, Ho PS, Groah S. The SCI Exercise Self-Efficacy Scale (ESES): development and psychometric properties. Int J Behav Nutr Phys Act 2007;4:34.

26. Van der Ploeg HP, Streppel KR, van der Beek AJ, van der Woude LH, Vollenbroek-Hutten M, van Mechelen W. The physical activity scale for individuals with physical disabilities: test-retest reliability and comparison with an accelerometer. J Phys Act Health 2007;4:96-100. 